ФГБОУ ВО Читинская государственная медицинская академия Минздрава России, Чита, Россия

Сахарный диабет 2 типа (СД2) ассоциирован с повышенным риском низкотравматичных переломов, при этом минеральная плотность костной ткани (МПК) не отличается или даже несколько превышает популяционную возрастную норму. Хрупкость костной ткани при СД2 обусловлена нарушением микроархитектоники кортикальной ткани. Трабекулярный костный индекс (ТКИ) дает косвенное представление о состоянии микроархитектоники костной ткани при рутинной двухэнергетической рентгеновской остеоденситометрии и обладает прогностическими свойствами в отношении низкотравматичных переломов. Представленный в статье клинический случай отражает современный взгляд на особенности диагностики и лечения остеопороза при СД2 с множественными осложнениями и наличием сопутствующей эндокринной патологии. Наличие низктравматического перелома, низкого ТКИ, высокого риска переломов, оцененного FRAX, низких показателей маркеров костного метаболизма и тенденции к гипокальциемии у пациентки с СД 2 типа послужили показанием для назначения анаболической терапии остеопороза - терипаратидом.

кЛючеВЫЕ СлОВА: Сахарный диабет 2 типа; остеопороз; трабекулярный костный индекс; терипаратид;:

\title{
TRABECULAR BONE SCORE FOR THE DIAGNOSTICS OF OSTEOPOROSIS IN SUBJECTS WITH TYPE 2 DIABETES MELLITUS: A CLINICAL CASE
}

\author{
(c) T.A. Grebennikova, Z.E. Belaya
}

Endocrinology Research Centre, Moscow, Russian Federation

Type 2 diabetes mellitus (T2DM) is associated with higher fracture risk but, better bone mineral density (BMD). Alteration of the skeletal material or microstructure may be an underlying mechanism for the discrepancy between BMD and fracture risk in diabetes. The trabecular bone score has been proposed as an indirect measurement of bone microarchitecture with the routine dual energy absorptiometry. We present a clinical case of diagnosis and treatment of osteoporosis associated with T2DM in patient with a low-trauma fracture and concomitant endocrine disorder.

KEYWORDS: type 2 diabetes mellitus; osteoporosis; trabecular bone score; teriparatide;

\section{АКТУАЛЬНОСТЬ}

СД2 предопределяет развитие множественных осложнений со стороны органов-мишеней, среди которых до недавнего времени не выделяли костную ткань. Установлено, что СД2 является независимым фактором риска переломов, не связанным с увеличением индекса массы тела и повышенной частотой падений [1, 2]. Данные эпидемиологических исследований показывают, что пожилые люди с СД2 имеют более высокий риск остеопоротических переломов, при этом риск переломов конечностей увеличивается на 50-80\% [3, 4].

Известно, что МПК определяет примерно 70\% общей прочности кости $[5,6]$, однако данный параметр у пациентов с СД2, как правило, выше общепопуляционного уровня. [7] Этот парадокс объясняется тем, что показатель МПК не учитывает другие факторы, влияющие на прочность кости, такие как трабекулярная микроархитектура, кортикальная макрогеометрия и скорость костного ремоделирования. [8-10]

Трабекулярный костный индекс (ТКИ) - это новый тканевой показатель, оценивающий пиксельные отклонения по шкале градаций серого на денситометрических изображениях поясничного отдела позвоночника [11].
ТКИ ассоциирован с микроархитектоникой костной ткани и является независимым параметром, определяющим риск низкотравматичных переломов вне зависимости от МПК [12-14]. В крупном когортном исследовании было показано, что у пациентов с сахарным диабетом 2 типа ТКИ имеет лучшее прогностическое значение в отношении переломов, чем МПК. [15]

Во всем мире для определения 10-летней вероятности риска переломов и необходимости начала антиостеопоротической терапии используется алгоритм $\operatorname{FRAX}[16,17,18]$, который, однако, не учитывает СД2 в качестве причины вторичного остеопороза и основывается на МПК, таким образом, недооценивая риск переломов в когорте больных с СД2. Тем не менее, модифицированный алгоритм FRAX, скорректированный по ТКИ, возможно использовать для оценки риска переломов у пациентов с вторичным остеопорозом, в том числе вследствие СД2.

Ряд экспертов предлагает выделить остеопороз, возникший вследствие СД2, в отдельную нозологическую единицу под названием «диабетопороз». Необходимость введения новой категории в классификацию вторичного остеопороза обусловлена особенностями патогенеза нарушения прочности кости при СД2, потребностью в определении четких критериев ранней диагностики 
остеопороза при данном заболевании для инициации медикаментозной терапии до возникновения низкотравматичных переломов. Согласно современным представлениям о заболевании можно предположить, что для диабетопороза характерны низкий костный обмен, нормальная МПК и низкий ТКИ. Кроме того, актуальным является вопрос выбора препарата при диабетопорозе, учитывая, что рандомизированные клинические исследования по оценке качества костной ткани на фоне лечения различными типами остеопоротических препаратов в данной когорте пациентов не проводились.

Следует обратить внимание, что у женщин в постменопаузе и мужчин старше 50 лет, страдающих СД2, по результатам денситометрии можно выявить снижение МПК, что, как описано выше, не характерно для диабетопороза. В этом случае патогенетически дефицит половых гормонов превалирует над глюкозотоксичностью в отношении костной ткани, поэтому такой вариант остеопороза имеет смысл лечить и оценивать как первичный остеопороз, осложненный СД2. Кроме того, иногда наблюдается развитие низкотравматичных переломов костей у пациентов с СД2 с нормальным костным обменом и показателями МПК и ТКИ, что свидетельствует о необходимости поиска как новых биомаркеров костного ремоделирования [19], так и новых методов оценки параметров костной ткани, влияющих на прочность костей.

В данной статье мы приводим описание диабетопороза на примере клинического случая пациентки с СД2, низкотравматичным переломом и сопутствующей эндокринной патологией.

\section{ОПИСАНИЕ СЛУЧАЯ}

Пациентка Л., 63 лет поступила в отделение нейроэндокринологии и остеопатий ФГБУ ЭНЦ с жалобами на боли в правой голени в покое и при ходьбе, частые падения (5 за год) и колебания уровня гликемии от 16 до 28 ммоль/л.

Из анамнеза известно, что сахарный диабет 2 типа диагностирован на фоне ожирения в 1999 году в возрасте 45 лет при профилактическом осмотре, гликемия при постановке заболевания - 7 ммоль/л. С 2003 года пациентка получала терапию пероральными сахароснижающими препаратами в различных сочетаниях, в том числе с инсулином пролонгированного действия. Самоконтроль гликемии был неудовлетворительный (1-2 раза в неделю), а с лета 2016 года уровень гликемии пациенткой не измерялся. Согласно медицинской документации, компенсации углеводного обмена на протяжении всего периода заболевания не отмечалось (гликированный гемоглобин в 2014-2016 годах $\mathrm{HbA}_{1 с}$ 8-8,5\%).

Из осложнений сахарного диабета в 2008 году диагностирована препролиферативная диабетическая ретинопатия, а в 2015 году пациентка перенесла лазерокоагуляцию сетчатки обоих глаз в связи с прогрессированием заболевания до пролиферативной ретинопатии. В 2014 году выявлена нейроишемическая форма синдрома диабетической стопы с развитием гнойно-некротических ран на поверхности 1 пальца правой стопы с последующей ампутацией 1 и 2 пальцев.

Также известно, что с 30 лет пациентка страдает гипертонической болезнью с максимальным подъемом артериального давления (АД) до 190/100 мм рт. ст. Кроме того,

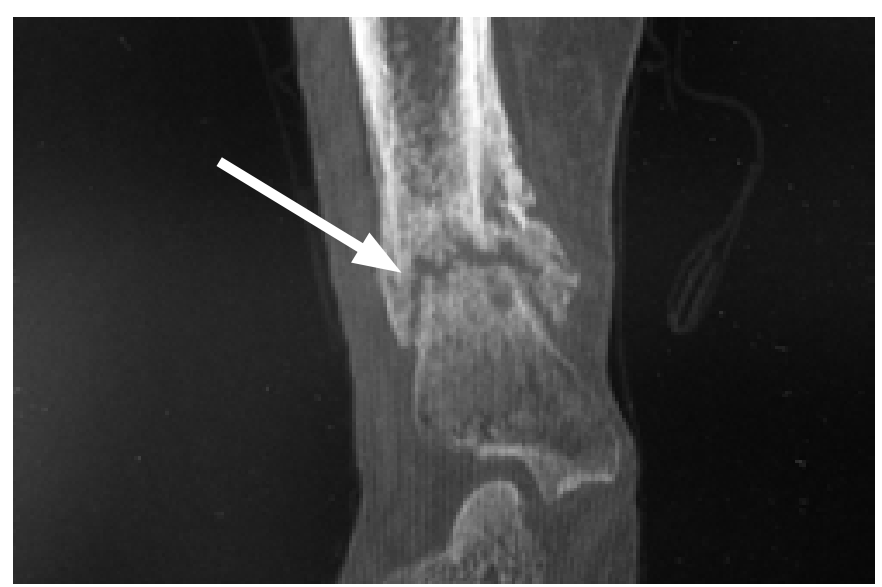

Рис. 1. МСКТ правой голени: перелом в нижней трети правой большеберцовой кости с признаками формирования костной мозоли.

в 2008 году пациентка перенесла верхнюю лобэктомию левого легкого в связи с диагностированным туберкулезом. В 2015 году - экстрафасциальная тиреоидэктомия по поводу папиллярного рака щитовидной железы, подтвержденного результатом гистологического исследования. В послеоперационном периоде принимала левотироксин натрия в заместительной дозе.

В июне 2016 года при падении с высоты собственного роста у пациентки развился перелом правой большеберцовой кости (рисунок 1), который в связи с образованием ложной мозоли в месте перелома привел к резкому ограничению подвижности пациентки. В течение 3-х месяцев после травмы пациентка передвигалась исключительно с помощью кресла-каталки. Возможность самостоятельного передвижения, опираясь на трость, появилась только в январе 2017 года спустя 7 месяцев после перелома. Лабораторное и инструментальное исследование фосфорно-кальциевого обмена и минеральной плотности костной ткани не проводилось.

При осмотре в апреле 2017 года: рост 173 см, вес 98 кг, ИМТ=32,7 кг/м². Отмечается хромота на правую ногу при ходьбе, передвижение самостоятельное с помощью трости, в пределах комнаты - без опоры.

При поступлении в стационар выявлена декомпенсация углеводного обмена - гликированный гемоглобин 11,6\%. Пациентка переведена на традиционную базис-болюсную инсулинотерапию инсулинами продленного и ультракороткого действия, и на фоне коррекции терапии уровень гликемии достиг индивидуальных целевых значений. Также было проведено комплексное обследование на предмет прогрессирования микрососудистых осложнений сахарного диабета 2 типа: подтверждена пролиферативная диабетическая ретинопатия обоих глаз, диабетической болезни почек не выявлено - СКФ 87мл/мин/1,73м³, анализ мочи на микроальбуминурию не проводился в связи с обострением хронического цистита.

Учитывая неконсолидированный перелом правой большеберцовой кости, выполнена мульти спиральная компьютерная томография (МСКТ) правой нижней конечности - отмечена умеренно положительная динамика за последние 2 месяца. По результатам ультразвукового дуплексного сканирования вен нижних конечностей патологических изменений и нарушений кровотока не выявлено. Пациентка осмотрена специалистом кабинета «Диабетическая стопа», рекомендована функциональная разгрузка правой нижней конечности, постоянное ношение голе- 
TBS reference graph TDE L1-L4: 1.186

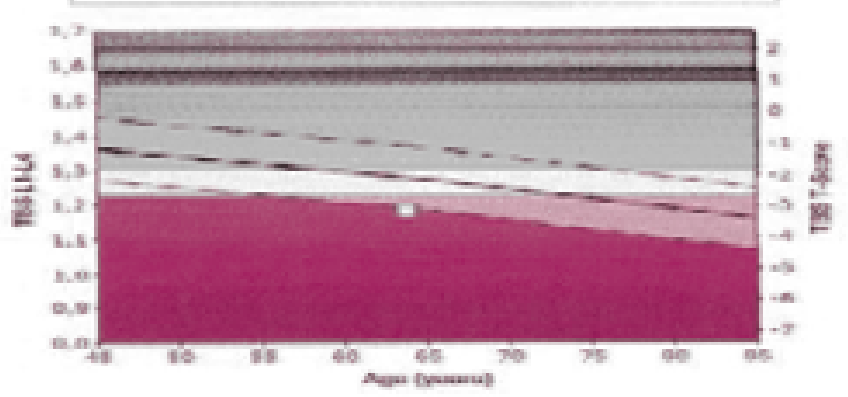

Additional results

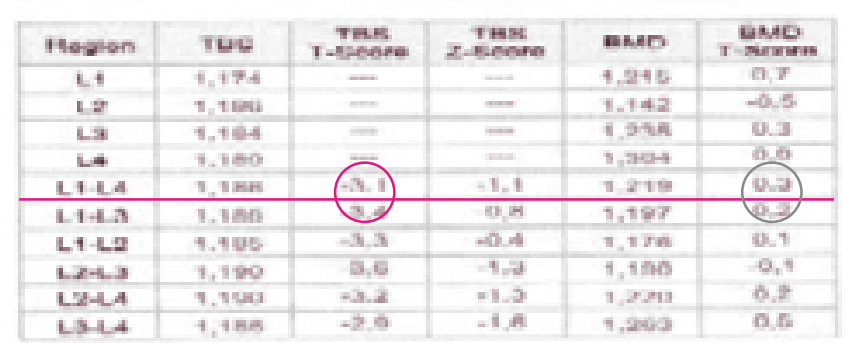

Рис. 2. Трабекулярный костный индекс пациентки Л.: несмотря на отсутствие снижения минеральной плотности кости в поясничных позвонках (Т-критерий 0.3), у больной имеется деградированная микроархитектоника с ТКИ 1.186 и Т-критерием -3.1 в той же зоне скелета (L1-L4).

ностопного ортеза в течение 2-3 месяцев до полной консолидации перелома.

Кроме того, в виду наличия первичного гипотиреоза в исходе тиреоидэктомии по поводу папиллярного рака щитовидной железы, пациентке проведено ультразвуковое исследование - данных за рецидив заболевания щитовидной железы не выявлено; уровень тиреоглобулина в пределах референсных значений. Отмечено повышение ТТГ выше референсных значений, в связи с чем была увеличена доза левотироксина натрия.

Одной из задач при обследовании данной пациентки с ранним развитием гипертонической болезни и ожирения, сахарным диабетом 2 типа и низкотравматичным переломом являлось исключение эндогенного гиперкортицизма. Было выявлено повышение свободного кортизола в слюне до 12,4 нмоль/л (0,5-9,4), при этом малая проба с 1 мг дексаметазона была положительной и уровень свободного кортизола в суточной моче не превышал референсных значений, поэтому согласно современным алгоритмам [20] эндогенный гиперкортицизм был исключен.

Из целого ряда тяжелых заболеваний, имеющихся у пациентки, доминирующим симптомом, резко ухудшающим качество жизни, являлся длительно неконсолидированный перелом правой большеберцовой кости. Поэтому важно было оценить состояние костной системы и минерального обмена, а также определить вероятность возникновения новых низкотравматичных переломов.

Для подсчета 10-летней вероятности риска переломов посредством алгоритма FRAX были собраны недостающие клинические данные, которые представлены в таблице 1. Учитывая наличие в анамнезе низкотравматичного перелома голени, были исключены компрессионные переломы тел позвонков с помощью проведения МСКТ позвоночного столба. По результатам денситометрии (DXA) снижения
Таблица 1. Факторы риска остеопороза пациентки Л

\begin{tabular}{lc}
\hline \multicolumn{1}{c}{ Фактор риска } & Наличие \\
\hline Предшествующий перелом & Да \\
Перелом бедра у матери & Да \\
Курение в настоящее время & Нет \\
Прием глюкокортикоидов & Нет \\
Ревматоидный артрит & Нет \\
Вторичный остеопороз & Нет \\
Ежедневный прием алкоголя более 3 единиц & Нет \\
\hline
\end{tabular}

'Сахарный диабет 1 типа, несовершенный остеогенез, длительно нелеченный гипертиреоз, гипогонадизм или ранняя менопауза (<45 лет), хроническое недоедание или мальабсорбция и хроническое заболевание печени

Таблица 2. МПК по результатам остеоденситометрии

\begin{tabular}{lc}
\hline \multicolumn{1}{c}{ Область исследования } & Т-критерий \\
\hline L1-L4 & 0,3 \\
Neck & $-0,4$ \\
Total hip & 0 \\
\hline
\end{tabular}

МПК в поясничных позвонках и шейке бедренной кости не было выявлено (таблица 2), однако отмечался низкий TKИ L1-L4 1,186 (рисунок 2), ассоциированный с высоким риском новых низкотравматичных переломов. В результате, при подсчете 10-летней вероятности риска переломов FRAX с учетом MПК и поправкой на показатель ТКИ риск перелома шейки бедра составил 0,6\%, а общих остеопоротических переломов - 25\%, что в совокупности с уже случившимся низкотравматичным переломом голени безусловно определяло необходимость начала антиостеопоротического лечения.

При лабораторном исследовании крови было выявлено снижение уровня маркеров костного ремоделирования (остеокальцина) и витамина D (таблица 3). Уровень кальция крови был приближен к нижней границе референсного интервала (таблица 3), а паратгормон снижен до 10,42 пг/мл (15-65), что позволило сделать вывод о наличии послеоперационного гипопаратиреоза в исходе тиреоидэктомии с сохранением компенсаторного синтеза паратгормона не удалёнными околощитовидными железами. Учитывая низкую скорость костного обмена, а также риск развития гипокальцемии, терапия бисфосфонатами или деносумабом была противопоказана. Анаболическая терапия терипаратидом (1-34 фрагментом паратгормона) являлась патогенетически оправданной и единственно возможной в данном клиническом случае.

До начала антиостеопоротической терапии был назначен колекальциферол в лечебной дозе для компенсации дефицита витамина D. После инициации терапии терипаратидом в дозе 20 мкг в сутки уровень остеокальцина через 10 дней терапии значимо не изменился (табл. 3), как наблюдается при лечении постменопаузального остепороза, т.к. нарушение костного ремоделирования при сахарном диабете 2 типа отличается низкой скоростью костного обмена и процесс выраженного увеличения костеобразования в первые месяцы терапии не ожидается. Пациентке было рекомендовано продолжить лечение терипаратидом 20 мкг/сут в сочетании с препаратами витамина D на мак- 
Таблица 3. Показатели фосфорно-кальциевого обмена

\begin{tabular}{lcc}
\hline \multicolumn{1}{c}{ Параметр } & Значение & Референсный интервал \\
\hline Кальций общий, ммоль/л & 2,21 & $2,2-2,55$ \\
Кальций ионизированный, ммоль/л & 1,06 & $1,03-1,29$ \\
Фосфор, ммоль/л & 1,35 & $0,74-1,52$ \\
Паратгормон, пг/мл & 10,42 & $15-65$ \\
Витамин D, нг/мл & 16 & $30-100$ \\
С-концевой телопептид коллагена 1 типа, нг/мл & 0,13 & $0,01-0,69$ \\
Остеокальцин, нг/мл & 7,29 & $11-43$ \\
Остеокальцин через 10 дней терапии терипаратидом, нг/мл & 6,67 & $11-43$ \\
\hline
\end{tabular}

симально возможный период, указанный в инструкции к терипаратиду, - 2 года.

\section{ОБСУЖДЕНИЕ}

Термин «Диабетопороз» был предложен профессором S Ferrari в 2015 году как особый тип нарушения качества костной ткани у пациентов с СД2. [21]. S Ferrari объясняет изменение микроахитектоники кости в условиях гипергликемии и инсулинорезистености прямым повреждающим действием конечных продуктов гликирования на коллагеновыесвязи, низкой скоростью костного ремоделирования, окислительным стрессом, а также взаимодействием между молекулярными механизмами костеообразования и регуляцией метаболизма глюкозы. В исследовании Dhaliwal впервые была показана корреляция между уровнем гликемического контроля и ТКИ. [22] Таким образом, при СД2 в качестве предиктора низкотравматичных переломов большую роль играет именно ТКИ, ассоциированный с микроархитектоникой костной ткани, что было подтверждено рядом исследований. $[15,23]$ Алгоритм FRAX, скорректированный по ТКИ, также лучше прогнозирует 10-летнюю вероятность переломов в когорте пациентов с СД2. [24]

Характерный для диабетопороза низкий костный обмен лабораторно проявляется в виде снижения маркеров костной резорбции (С-концевой телопептид коллагена 1 типа) [25] и костного синтеза (костноспецифическая щелочная фосфатаза, остеокальцин, пропептид коллагена 1 типа). [26-28]. Интересно, что и уровень паратгормона при данном заболевании снижен на 20-50\% [25-27]. Низкая скорость костного ремоделирования рассматривается некоторыми исследователями как независимый фактор риска низкотравматичных переломов. [27, 29]

Пациенты с СД2 имеют не только повышенный риск переломов, но также склонны к нарушению консолидации перелома [30], что было продемонстрировано в представленном клиническом случае. Как правило, ожидается, что в месте перелома у здоровых людей скорость костного ремоделирования увеличится. Однако при диабетопорозе при изначально низком костном обмене активность остеокластов возрастает незначительно, что приводит к менее эффективному удалению поврежденной кости и хряща, образующегося при эндохондральной оссификации. Как следствие ухудшается локальная стимуляция формирования новой кости и образуется ложная костная мозоль. [30].
Экспериментальное исследование на грызунах с СД и переломом бедра показало, что введение препарата паратгормона способно снизить неблагоприятные эффекты СД на костный обмен. [31]

Антирезорбтивные препараты (бисфосфонаты, деносумаб) и анаболическая терапия (терипаратид) однозначно доказали свою эффективность при лечении постменопаузального и сенильного остеопороза [32-34], однако исследование применения терапии остеопороза при диабетопорозе не проводилось. Возможно, ввиду подавленного костеобразования и общего снижения костного ремоделирования, а также нарушения образования костной мозоли в данном случае анаболическая терапия терипаратидом является предпочтительней. [35] Интересным представляется использование при диабетопорозе принципиально новых лекарственных препаратов (антител к склеростину), которые, кроме мощного анаболического эффекта через стимуляцию канонического Wnt-сигнального пути, обладают и антирезорбтивным действием. [36] Данные препараты (ромосозумаб и блосозумаб) находятся в различных фазах клинических исследований.

\section{ЗАКЛЮЧЕНИЕ}

Ввиду повышенного риска низкотравматичных переломов при СД2 и низкой чувствительностью существующих диагностических методов (FRAX, DXA) для данной категории пациентов необходимо внедрение новых методов ранней диагностики лиц с СД2 с повышенной склонностью к переломам с более широким внедрением трабекулярного костного индекса (ТКИ) при применении рутинной денситометрии и введением этого показателя в алгоритм FRAX. Необходимы также дальнейшие исследования по оценке эффективности различных методов лечения остеопороза при СД2 с учетом специфических патофизиологических изменений костной ткани при этом заболевании.

\section{ДОПОЛНИТЕЛЬНАЯ ИНФОРМАЦИЯ}

Согласие пациента. Пациент добровольно подписал информированное согласие на публикацию персональной медицинской информации в обезличенной форме в журнале «Остеопороз и остеопатии».

Конфликт интересов. Авторы декларируют отсутствие явных и потенциальных конфликтов интересов, связанных с публикацией настоящей статьи. 


\section{СПИСОК ЛИТЕРАТУРЫ | REFERENCES}

1. Leslie WD, Rubin MR, Schwartz AV, Kanis JA. Type 2 diabetes and bone. J Bone Miner Res. 2012;27(11):2231-2237. doi: 10.1002/jbmr.1759

2. Ялочкина Т.О., Белая Ж.Е., Рожинская Л.Я., и др. Переломы костей при сахарном диабете 2 типа: распространенность и факторы риска // Сахарный диабет. - 2016. - Т. 19. - №5. - С. 359-365. [Yalochkina TO, Belaya JE, Rozhinskaya LY, et al. Bone fractures in patients with type 2 diabetes mellitus: prevalence and risk factors. Diabetes mellitus. 2016;19(5):359-365. (In Russ.)] doi: 10.14341/dm7796

3. Schwartz AV, Sellmeyer DE, Ensrud KE, et al. Older Women with Diabetes Have an Increased Risk of Fracture: A Prospective Study. J Clin Endocr Metab. 2001;86(1):32-38. doi: 10.1210/jcem.86.1.7139

4. Bonds DE, Larson JC, Schwartz AV, et al. Risk of Fracture in Women with Type 2 Diabetes: the Women's Health Initiative Observational Study. J Clin Endocr Metab. 2006;91(9):3404-3410. doi: 10.1210/jc.2006-0614

5. NIH Consensus Development Panel on Osteoporosis Prevention, Diagnosis, and Therapy. Osteoporosis Prevention, Diagnosis, and Therapy. JAMA. 2001;285(6):785-795. doi:10.1001/jama.285.6.785

6. Kanis JA, McCloskey EV, Johansson H, et al. European guidance for the diagnosis and management of osteoporosis in postmenopausal women. Osteoporos Int. 2012;24(1):23-57. doi: 10.1007/s00198-012-2074-y

7. Vestergaard P. Discrepancies in bone mineral density and fracture risk in patients with type 1 and type 2 diabetes-a meta-analysis. Osteoporos Int. 2006;18(4):427-444. doi: 10.1007/s00198-006-0253-4

8. Brandi ML. Microarchitecture, the key to bone quality. Rheumatology. 2009;48(suppl 4):iv3-iv8. doi: 10.1093/rheumatology/kep273

9. Ulivieri FM, Silva BC, Sardanelli F, et al. Utility of the trabecular bone score (TBS) in secondary osteoporosis. Endocrine. 2014;47(2):435-448. doi: 10.1007/s12020-014-0280-4.

10. Link TM, Majumdar S. Current diagnostic techniques in the evaluation of bone architecture. Current Osteoporosis Reports. 2004;2(2):47-52. doi: 10.1007/s11914-004-0003-5

11. Ц Цориев Т.Т., Белая Ж.Е., Мельниченко Г.А. Трабекулярный костный индекс-неинвазивный метод оценки качества костной ткани на основании рутинной двухэнергетической денситометрии. Перспективы использования в клинической практике // Альманах клинической медицины. - 2016. - Т. 44. - №. 4. - С. 462-476. [Tsoriev TT, Belaya ZE, Mel'nichenko GA. Trabecular Bone Score A non-Invasive Analytical Method to Evaluate Bone Quality Based on Routine Dual-Energy Absorptiometry. Perspectives of Its Use in Clinical Practice. Almanac of Clinical Medicine. 2016;44(4):462-476. (In Russ.)] doi: 10.18786/2072-0505-2016-44-4-462-476

12. Bousson $V$, Bergot $C$, Sutter B, et al. Trabecular bone score (TBS): available knowledge, clinical relevance, and future prospects. Osteoporos Int. 2011;23(5):1489-1501. doi: 10.1007/s00198-011-1824-6

13. Roux JP, Wegrzyn J, Boutroy S, et al. The predictive value of trabecular bone score (TBS) on whole lumbar vertebrae mechanics: an ex vivo study. Osteoporos Int. 2013;24(9):2455-2460. doi: 10.1007/s00198-013-2316-7

14. Pothuaud L, Carceller P, Hans D. Correlations between grey-level variations in $2 \mathrm{D}$ projection images (TBS) and 3D microarchitecture: Applications in the study of human trabecular bone microarchitecture. Bone. 2008;42(4):775-787. doi: 10.1016/j.bone.2007.11.018

15. Leslie WD, Aubry-Rozier B, Lamy O, Hans D. TBS (Trabecular Bone Score) and Diabetes-Related Fracture Risk. J Clin Endocr Metab. 2013:98(2):602-609. doi: 10.1210/jc.2012-3118

16. Kanis JA, Johansson $\mathrm{H}$, Oden $\mathrm{A}$, et al. Worldwide uptake of FRAX. Archives of Osteoporosis. 2014;9(1). doi: 10.1007/s11657-013-0166-8

17. McCloskey EV, Odén A, Harvey NC, et al. Adjusting Fracture Probability by Trabecular Bone Score. Calcif Tissue Int. 2015;96(6):500-509. doi: 10.1007/s00223-015-9980-x

18. Мельниченко Г.А., Белая Ж.Е., Рожинская Л.Я., и др. Краткое изложение клинических рекомендаций по диагностике и лечению остеопороза российской ассоциации эндокринологов // Остеопороз и остеопатии. - 2016. - Т. 19. - №3. - С. 28-36. [Melnichenko GA, Belaya ZE, Rozhinskaya LY, et al. Summary of Clinical Guidelines for the Diagnosis and Treatment of Osteoporosis of the Russian Association of Endocrinologists. Osteoporosis and Bone Diseases. 2016;19(3):28-36. (In Russ.)] doi: 10.14341/osteo2016328-36

19. Гребенникова Т.А., Белая Ж.Е., Рожинская Л.Я., и др. Эпигенетические аспекты остеопороза // Вестник Российской академии ме- дицинских наук. - 2015. - Т. 70. - №. 5. [Grebennikova TA, Belaya ZE, Rozhinskaya LY, et al. Epigenetic Aspects of Osteoporosis. Annals of the Russian academy of medical sciences. 2015;70(5):541. (In Russ.)] doi: 10.15690/vramn.v70.15.1440

20. Мельниченко Г.А., Дедов И.И., Белая Ж.Е., и др. Болезнь Иценко-Кушинга: клиника, диагностика, дифференциальная диагностика, методы лечения // Проблемы Эндокринологии. - 2015. - Т. 61. №2. - C. 55-77. [Melnichenko GA, Dedov II, Belaya ZE, et al. Cushing's disease: the clinical features, diagnostics, differential diagnostics, and methods of treatment. Problems of Endocrinology. 2015;61(2):55. (In Russ.)] 10.14341/probl201561255-77

21. Ferrari S. Pathophysiology of diabetoporosis. Endocrine Abstracts. 2015. doi: 10.1530/endoabs.37.S17.2

22. Dhaliwal R, Cibula D, Ghosh C, et al. Bone quality assessment in type 2 diabetes mellitus. Osteoporos Int. 2014;25(7):1969-1973. doi: 10.1007/s00198-014-2704-7

23. Hans D, Goertzen AL, Krieg M-A, Leslie WD. Bone microarchitecture assessed by TBS predicts osteoporotic fractures independent of bone density: The manitoba study. J Bone Miner Res. 2011;26(11):2762-2769. doi: 10.1002/jbmr.499

24. Choi YJ, Ock SY, Chung Y-S. Trabecular Bone Score (TBS) and TBS-Adjusted Fracture Risk Assessment Tool are Potential Supplementary Tools for the Discrimination of Morphometric Vertebral Fractures in Postmenopausal Women With Type 2 Diabetes. J Clin Densitom. 2016;19(4):507-514. doi: 10.1016/j.jocd.2016.04.001

25. Dobnig H, Piswanger-Sölkner JC, Roth M, et al. Type 2 Diabetes Mellitus in Nursing Home Patients: Effects on Bone Turnover, Bone Mass, and Fracture Risk. J Clin Endocr Metab. 2006;91(9):3355-3363. doi: 10.1210/jc.2006-0460

26. Ardawi M-SM, Akhbar DH, AlShaikh A, et al. Increased serum sclerostin and decreased serum IGF-1 are associated with vertebral fractures among postmenopausal women with type-2 diabetes. Bone. 2013;56(2):355-362. doi: 10.1016/j.bone.2013.06.029

27. Yamamoto M, Yamaguchi T, Nawata K, et al. Decreased PTH Levels Accompanied by Low Bone Formation Are Associated with Vertebral Fractures in Postmenopausal Women with Type 2 Diabetes. J Clin Endocr Metab. 2012;97(4):1277-1284. doi: 10.1210/jc.2011-2537

28. Gaudio A, Privitera F, Battaglia K, et al. Sclerostin Levels Associated with Inhibition of the Wnt/B-Catenin Signaling and Reduced Bone Turnover in Type 2 Diabetes Mellitus. J Clin Endocr Metab. 2012;97(10):3744-3750. doi: 10.1210/jc.2012-1901

29. Rubin MR, Patsch JM. Assessment of bone turnover and bone quality in type 2 diabetic bone disease: current concepts and future directions. Bone Research. 2016;4:16001. doi: 10.1038/boneres.2016.1

30. Blakytny R, Spraul M, Jude EB. Review: The Diabetic Bone: A Cellular and Molecular Perspective. The International Journal of Lower Extremity Wounds. 2011;10(1):16-32. doi: 10.1177/1534734611400256

31. Hamann C, Picke A-K, Campbell GM, et al. Effects of Parathyroid Hormone on Bone Mass, Bone Strength, and Bone Regeneration in Male Rats With Type 2 Diabetes Mellitus. Endocrinology. 2014;155(4):1197-1206. doi: 10.1210/en.2013-1960

32. Белая ЖЕ, Рожинская ЛЯ, Мельниченко ГА. Ибандронат (Бонвива) - новые возможности в лечении остеонороза: повышение приверженности к терапии - оптимизация исходов лечения // Остеопороз и остеопатии. - 2006. - T. 9. - №3. - С. 23-30. [Belaya ZE, Rozhinskaya LY, Mel'nichenko GA. Ibandronat (Bonviva) - novye vozmozhnosti v lechenii osteonoroza: povyshenie priverzhennosti k terapii - optimizatsiya iskhodov lecheniya. Osteoporosis and Bone Diseases. 2006;9(3):23-30. (In Russ.)] doi: 10.14341/osteo2006323-30

33. Белая Ж.Е., Рожинская Л.Я. Новые направления в терапии остеопороза - применение моноклональных человеческих антител к RANKL (Деносумаб). // Остеопороз и остеопатии. 2011. - T. 14. - №2. - C. 23-26. [Belaya ZE, Rozhinskaya LY. Novye napravleniya $v$ terapii osteoporoza - primenenie monoklonal'nykh chelovecheskikh antitel k RANKL (Denosumab). Osteoporosis and Bone Diseases. 2011;14(2):23-26. (In Russ.)] doi: 10.14341/osteo2011223-26

34. Белая Ж.Е., Рожинская Л.Я. Анаболическая терапия остеопороза. Терипапаратид: эффективность, безопасность и область применения // Остеопороз и остеопатии. - 2013. - Т. 16. - №2. - С. 32-40. [Belaya ZE, Rozhinskaya LY. Anabolicheskaya terapiya osteoporoza. Teripaparatid: effektivnost', bezopasnost' i oblast' primeneniya. Osteoporosis and Bone Diseases. 2013;16(2):32-40. (In Russ.)] 
35. Gonnelli S, Caffarelli C, Giordano N, Nuti R. The prevention of fragility fractures in diabetic patients. Aging Clin Exp Res. 2014;27(2):115-124. doi: 10.1007/s40520-014-0258-3

36. Гребенникова Т.А., Белая Ж.Е., Рожинская Л.Я., Мельниченко Г.А. Канонический сигнальный путь Wnt/ß-катенин: от истории открытия до клинического применения. // Терапевтический архив. - 2016. - T. 88. - №. 10. - C. 74-81. [Grebennikova TA, Belaya ZE, Rozhinskaya LY, Melnichenko GA. The canonical Wnt/ $\beta$-catenin pathway: From the history of its discovery to clinical application. Ter Arkh. 2016;88(10):74. (In Russ.)] doi: 10.17116/terarkh201688674-81

\section{ИНФОРМАЦИЯ ОБ АВТОРАХ [AUTHORS INFO]}

Гребенникова Татьяна Алексеевна [Tatiana A. Grebennikova]; адрес: Россия, 117036, Москва, ул. Дм. Ульянова, д. 11 [address: 11 Dm.Ulyanova street, 117036 Moscow, Russia] телефон: +79854831694; ORCID: http://orcid.org/0000-0003-1413-1549; eLibrary SPIN: 4380-5447; e-mail: grebennikova@hotmail.com Белая Жанна Евгеньевна, д.м.н., професcop [Zhanna E. Belaya, MD, PhD, Professor]; e-mail: jannabelaya@gmail.com; ORCID: http://orcid.org/0000-0002-66746441; eLibrary SPIN: 4746-7173.

\section{ЦИТИРОВАТЬ:}

Гребенникова Т.А., Белая Ж.Е. Трабекулярный костный индекс для диагностики остеопороза при сахарном диабете 2 типа: клинический случай. // Остеопороз и остеопатии. — 2017. — T. 20. — №1. — C.22-27. doi: 10.14341/osteo2017139-43

\section{TO CITE THIS ARTICLE:}

Grebennikova TA, Belaya ZE. Trabecular bone score for the diagnostics of osteoporosis in subjects with type 2 diabetes mellitus: a clinical case. Osteoporosis and bone diseases. 2017;20(1):22-27. doi: 10.14341/osteo2017139-43 